\title{
Study of the development of fetal baboon brain using magnetic resonance imaging at 3 Tesla
}

\author{
Feng Liu ${ }^{a}, c^{,}{ }^{*}$, Marianne Garland ${ }^{b}$, Yunsuo Duan ${ }^{a, c}$, Raymond I. Stark ${ }^{b}$, Dongrong Xu $^{\mathrm{a}, \mathrm{c}}$, \\ Zhengchao Dong ${ }^{a, c}$, Ravi Bansala,c, Bradley S. Peterson ${ }^{a, c}$, and Alayar Kangarlua,c \\ aDepartment of Psychiatry, Columbia University Medical Center, New York, NY 10032, USA \\ bDepartment of Pediatrics, Columbia University Medical Center, New York, NY 10032, USA \\ cNew York State Psychiatric Institute, New York, NY 10032, USA
}

\begin{abstract}
Direct observational data on the development of the brains of human and nonhuman primates is on remarkably scant, and most of our understanding of primate brain development is extrapolated from findings in rodent models. Magnetic resonance imaging (MRI) is a promising tool for the noninvasive, longitudinal study of the developing primate brain. We devised a protocol to scan pregnant baboons serially at $3 \mathrm{~T}$ for up to $3 \mathrm{~h}$ per session. Seven baboons were scanned 1-6 times, beginning as early as 56 days post-conceptional age, and as late as 185 days (term $\sim 185$ days). Successful scanning of the fetal baboon required careful animal preparation and anesthesia, in addition to optimization of the scanning protocol. We successfully acquired maps of relaxation times ( $T_{1}$ and $T_{2}$ ) and high-resolution anatomical images of the brains of fetal baboons at multiple time points during the course of gestation. These images demonstrated the convergence of gray and white matter contrast near term, and furthermore demonstrated that the loss of contrast at that age is a consequence of the continuous change in relaxation times during fetal brain development. These data furthermore demonstrate that maps of relaxation times have clear advantages over the relaxation time weighted images for the tracking of the changes in brain structure during fetal development. This protocol for in utero MRI of fetal baboon brains will help to advance the use of nonhuman primate models to study fetal brain development longitudinally.
\end{abstract}

\section{Keywords}

Fetal baboon; MRI; Brain development; 3 T; Relaxation times; $T_{1} ; T_{2}$

\section{Introduction}

The development of the central nervous system (CNS) in primates during fetal and early postnatal life involves highly orchestrated changes in its structural and biochemical

\footnotetext{
* Corresponding author. New York State Psychiatric Institute, 1051 Riverside Drive, Unit 74, New York, NY 10032, USA. Fax: +1 212 543 0522. E-mail address: liuf@ childpsych.columbia.edu (F. Liu).

Available online on ScienceDirect (www.sciencedirect.com)

Publisher's Disclaimer: This article was published in an Elsevier journal. The attached copy is furnished to the author for non-commercial research and education use, including for instruction at the author's institution, sharing with colleagues and providing to institution administration. Other uses, including reproduction and distribution, or selling or licensing copies, or posting to personal, institutional or third party websites are prohibited. In most cases authors are permitted to post their version of the article (e.g. in Word or Tex form) to their personal website or institutional repository. Authors requiring further information regarding Elsevier's archiving and manuscript policies are encouraged to visit: http://www.elsevier.com/copyright
} 
characteristics of the brain (Black et al., 2004; Dobbing and Sands, 1973; Levitt, 2003). As a consequence of the limitations in neurobiological research using fetal human and nonhuman primates, substantial gaps exist in our knowledge of early CNS development. Over-reliance on extrapolation from more detailed studies of fetal brain development in rodents may have limited relevance for, and may even confound, our understanding of brain development in primates (Levitt, 2003). Thus, defining the ontogeny of brain development in the normal fetus of nonhuman primates is vitally important for improving our understanding of the origins of neurological and psychiatric health and disease across the lifespan (Dawson et al., 2000).

Most of our knowledge of fetal brain development in primates has come from post-mortem histological studies that examine cellular, synaptic, and molecular events during development (Huttenlocher, 1979, 1990; Huttenlocher and Dabholkar, 1997). The scientific value of these studies is severely limited, however, by their cross-sectional nature, by the variability of the quality of tissue and characteristics of the study samples, and by the expense required to study more than a small set of brain regions. Postmortem histological analyses, for example, require detailed volumetric and morphometric measurements to obtain data for a single brain region at a single time point in development (Benes and Lange, 2001; Blinkov and Glezer, 1968; Dekaban, 1977; Huttenlocher, 1990; Lewis, 2002; West, 1999). The tissue used in these studies, moreover, is often compromised by the effects of postmortem fixation that introduces artifact into volumetric measures (Blinkov and Glezer, 1968; Dekaban, 1977). In addition, because the detailed analyses are typically limited to a small fraction of the brain, they fail to provide information about the entire brain, which is needed to understand the regional specificity of brain maturation (Benes and Lange, 2001; West, 1999). Finally, post-mortem analyses necessarily can provide information about the brain only in temporal cross-section, limiting the statistical power to discern developmental changes and severely impairing our ability to make valid inferences concerning development across time (Kraemer et al., 2000).

Investigation of fetal brain development in vivo using magnetic resonance imaging (MRI) offers an attractive alternative to postmortem studies for developmental studies, particularly for longitudinal studies of fetal brain across gestation in individual pregnancies. Although MRI at present cannot provide information about the brain on the cellular level, it can provide details on the volumes and shapes of individual anatomical structures, white matter connectivity, and metabolism (Inder and Huppi, 2000). The application of MRI to the study of fetal brain development in humans, however, has been limited by concerns of safety for the developing human fetus (Elster, 1994; Myers et al., 1998). Given the similarity of brain structure across primate species, longitudinal studies of fetal brain development using nonhuman primates offers the attractive opportunity to advance our knowledge of brain development in a species that provides information of much greater relevance for our knowledge of human fetal development than do rodent studies (Levitt, 2003).

MRI of the fetal brain in situ within pregnant nonhuman primates, however, presents several challenges that until recently have proved intractable. These difficulties include the frequent artifacts in the images caused by maternal or fetal movements, the low signal-to-noise ratios (SNRs) associated with intra-abdominal imaging, the small size of the fetal brain, and the need for high spatial resolution to provide detailed images of the small fetal brain. These problems have undermined the use of in utero fetal MRI as a viable research tool.

We have exploited the advantages of high-field MRI, as well as other recent advances in imaging technologies, to address these longstanding difficulties. For instance, we have used higher field strength to improve the resolution and SNR of fetal brain images. The combination of improved slew rates, faster imaging sequences, and appropriate use of anesthesia all reduce or eliminate motion artifact. We have also addressed the deleterious effects on image quality caused by fluid flow, fat, and the interface between multiple tissues in the abdominal cavity. 
Finally, we have examined strategies for the design and selection of radiofrequency coils that affect the rate of energy deposition and other practical problems that can limit the feasibility of fetal imaging.

This report therefore details the development and validation of an investigative protocol for MR imaging of the fetal brain in pregnant baboons. Our final protocol includes procedures for anesthesia, specifications for radiofrequency coils, and details of pulse sequences that optimize image quality for multiple MRI modalities, including $T_{1^{-}}$and $T_{2}$-weighted anatomical MRI, relaxometry, and diffusion tensor imaging (DTI). The goal of these studies was to demonstrate the feasibility and utility of using MRI for longitudinal investigations of fetal brain development.

\section{Methods}

\section{Animals}

A colony of individually caged female and male baboons (Papio papio) was maintained in the Institute of Comparative Medicine of Columbia University. All animal care and experimental procedures were reviewed and approved by the IACUC of Columbia University and the New York State Psychiatric Institute. The study adhered to the guidelines of the American Association for Accreditation of Laboratory Animal Care and the U.S. Department of Agriculture.

Male baboons of proven fertility were maintained in large cages appropriate for breeding. Female baboons were housed in adjacent standard cages. Their general behavior, tumescent and menstrual cycles, reactions to neighbors and responses to procedures for environmental enrichment were monitored to evaluate acceptance in the colony. When accepted, females were placed with a male during the receptive phase of their cycle for a period of up to 5 days. Animals were then returned to their home cage and observed for signs of pregnancy. With the absence of changes in sex skin tumescence and menses at 30-45 days after breeding, an animal was assumed to have conceived and was assigned to a specific investigational protocol.

In preparation for MRI scanning, food but not water was withheld overnight and in the morning. Following sedation with ketamine $(5-10 \mathrm{mg} / \mathrm{kg}$ ) and atropine $(0.02-0.05 \mathrm{mg} / \mathrm{kg}, \mathrm{IM})$, pregnant baboons were removed from their home cages. They were weighed, and a uterine ultrasound was performed to assess the location of the placenta, to determine fetal orientation, and to measure heart rate. An intravenous line was placed for infusion of ringers lactate and D5W at rates of $60-120 \mathrm{ml} / \mathrm{h}$. The animal was intubated with a cuffed endotracheal tube. A urinary catheter was placed and urine was collected into a bag to keep the bladder empty and to prevent soiling in the MRI facility. Earplugs were placed in the external ear canal. The animal was then swaddled in blankets and a protective outer plastic wrap to facilitate thermoregulation and handling and to minimize MRI suite contamination. These procedures required a maximum of 15-20 min. The animal was then transferred to the MRI facilities in an adjacent building with use of an isolation transfer cage. For return transportation to the colony, the animal was weaned from inhaled anesthesia (below), extubated, and with establishment of regular respiration, was returned to the colony. Post-transport care included continuous observation for signs of return to full consciousness, and intermittent (at least hourly) observation for acceptance of special treats and fruit drinks that were offered. The total duration of food deprivation was 14-16 h, during which fluid, electrolytes, and glucose were maintained by IV supplement. The total duration of sedation and anesthesia ranged from 4 to $5.5 \mathrm{~h}$, of which 3 to $4.5 \mathrm{~h}$ were available for scanning.

Fetal and maternal motion initially was found to degrade severely the quality of fetal brain images. We subsequently found that carefully calibrated anesthesia increased the quality of 
images and enabled longer scans that were less affected by motion artifact. An MRI-compliant ventilator and anesthesia apparatus was used together with continuous monitoring of maternal heart rate, respiratory rate, end tidal $\mathrm{CO}_{2}$, pulse oximetry, and intermittent monitoring of blood pressure and temperature. We administered isoflurane (1.0\% to $3.0 \%$ ) during spontaneous breathing in our initial studies of fetal brain, but the contraction of maternal abdominal muscles during spontaneous breathing produced considerable movement artifact. This was overcome through use of an MRI-compatible ventilator, together with a greater depth of anesthesia, to override maternal breathing. The addition of nitrous oxide in a 40:60 mixture of $\mathrm{O}_{2}$ allowed us to reduce the concentration of isoflurane in the anesthetic regimen from $1.25-0.75 \%$ to $0.75-$ $0.25 \%$, while continuing to provide maternal and fetal quiescence. Anesthetic conditions were assessed and adjusted with information from continuous monitoring of maternal end expiratory $p \mathrm{CO}_{2}$, and $\mathrm{O}_{2}$ saturation, as well as heart and respiratory rates. Monitoring the respiratory pattern was particularly useful in detecting the occurrence of spontaneous breathing. This regimen allowed us to sustain the mother in a physiologically stable condition. When the mother and fetus were maintained under these anesthetic conditions throughout the scanning procedure, we found that all pulse sequences could be completed with minimal movement artifact.

Although motion artifact was reduced greatly through the use of anesthesia, artifacts caused by small fetal movements and by maternal respiration were to some degree inevitable. In instances where an artifact was known to have occurred, the scan was repeated. Respiratory gating of image acquisition was available with our ventilatory system, but its use did not improve image quality substantially.

The bladder of the baboon mother was kept empty during the scan, because a full bladder pressing against the fetal head can cause greater motion artifact with maternal breathing. In addition, the strong water signal in a full bladder produced a sizable inhomogeneity of signal intensity across the fetal brain (Fig. 1), likely the consequence of unwanted eddy currents in the electrically conductive urine. We therefore catheterized the pregnant mother to ensure that the bladder was empty.

A total of 7 pregnant baboons were studied. Two baboons were used to establish animal transport and care procedures within the MRI scanning environment. The other 5 pregnant baboons underwent repeated scans beginning at 56 days gestation (dg) (Table 1), with term gestation in our colony being 175-185 dg. In 2 pregnancies (\#434 and \#797), fetuses were scanned 5 to 6 times from the 2 nd trimester until term birth. In the other 3 pregnancies, scanning studies were limited to the 2 nd trimester because of other research protocols. The interval between scans was 2-3 weeks. We also performed follow-up scans on one fetal baboon (\#415) after it was born.

\section{MRI scanner}

MRI pulse sequences were implemented on a GE Signa EXCITE 3T/94 cm whole-body scanner equipped with the E2M4 version of LX software (GE Medical Systems, Milwaukee, WI). The system can achieve a gradient strength of $40 \mathrm{mT} / \mathrm{m}$, with a slew rate of $150 \mathrm{~T} / \mathrm{m} / \mathrm{s}$.

\section{Radiofrequency coils}

The primary challenge for selection of a radiofrequency (RF) coil for studies during pregnancy is the need for a sufficiently large coil to accommodate the pregnant baboon until term, yet sufficiently small to maximize SNR. Either a body coil or a phase-arrayed surface coil typically is used for scanning the human fetus (Huisman et al., 2002; Prayer et al., 2006). A body coil in transmitter and receiver mode provided inadequate SNR (Fig. 2, left) to resolve small 
structures in the brains of fetal baboons. This poor SNR was a consequence primarily of the distance between the fetal brain and the wall of the body coil.

Lacking an appropriate phase-arrayed surface coil, we next tried a head coil-based design with a sufficiently enlarged bore to accommodate a pregnant baboon. The SNR of the images improved but remained unsatisfactory. We then adopted use of a quadrature head coil manufactured by GE $(\sim 40 \mathrm{~cm}$ long, inner diameter $\sim 25 \mathrm{~cm})$ for subsequent scans of the abdomen of the pregnant baboon.

This GE head coil accommodated the pregnant baboon and provided an image quality superior to that of the other coils we tested (Fig. 2, right). With both ends of the GE head coil open, a pregnant baboon at term, equipped with thermal wrapping, sensors to monitor vital signs, a bladder catheter, and ventilatory tubing for administration of anesthesia, fit comfortably within the coil. Pregnant baboons were placed in the coil feet first.

\section{Specific absorption rate}

Of concern with fetal MRI is that the RF deposition during the scan may increase body temperature beyond the limits of safety to the fetus (Hand et al., 2006). Specific absorption rate (SAR), a measure of the rate of energy deposition to a subject, must be monitored carefully during high-field MRI scanning ( 3 T or greater), because of the greater RF power used at higher field strengths. We used RF-intense sequences, such as fast spin echo (FSE), for relaxometry and anatomical MRI, and therefore we interspersed RF-intense and non RF-intense sequences to reduce this thermal risk.

\section{In utero environment}

The fetal head is surrounded by amniotic fluid and numerous tissue layers, which together can induce severe phase-wrapping artifacts. When a large amount of tissue is outside the selected field of view (FOV), the signal from that tissue can be phase-wrapped into the FOV (Fig. 3, left). This is especially true for fluids and fatty tissues outside the FOV, as they have high signal intensities in some pulse sequences. Some sequences, such as FSE, are compatible with the scanner option "no phase wrap" (NPW) that suppresses these wrap-around artifacts, but at the expense of increased scan time (Fig. 3, right). Some other sequences, such as echo planar imaging (EPI), are not compatible with NPW. We therefore used a relatively large FOV in these EPI sequences to reduce phase-wrapping artifacts whenever they affected visualization of regions of interest (ROIs) within the image. Use of saturation bands to suppress signal from outside the selected FOV can also reduce phase wrapping. Saturation bands, however, can partially saturate signal from inside the selected FOV as well, thereby compromising SNR and contrast (Fig. 4). For this reason, we opted not to use saturation bands.

\section{Position and localization}

Positioning the fetal head within the isocenter of the magnet, where the magnetic field is most homogeneous, and then accurately localizing the head during image acquisition, is essential for obtaining images of optimal quality. The pregnant baboon was positioned feet-first in the head-coil in the left lateral position for easy access to ventilation tubing and physiological monitoring equipment, for improving the animal's comfort, and for reducing obstruction of the inferior vena cava. The position of the pregnant baboon in the coil was adjusted such that the head of the fetus was located as close as possible to the center of the RF coil. With a landmark at the center of the coil, a 3-plane localization sequence was used to locate coarsely the entire fetus. If the fetal brain was not centered in the magnet, the mother's position was adjusted in the coil. In some instances, an experienced perinatologist adjusted the position of the fetus in the uterus to optimize head positioning and to reduce motion artifact associated with either movement or maternal breathing (Fig. 5). 
Every attempt was made to obtain images in the true "axial" plane, because axial images are optimal for evaluation of sulcation and gyration of the fetal brain, features that indicate stages of brain maturation. Axial images are also useful for assessment of the degree of symmetry in brain development, and they are readily compared with data in many clinical studies acquired in the same plane. A typical 3-plane localizer sequence with a 36-cm FOV and slice thickness of $5 \mathrm{~mm}$ identified the location the fetal brain. A single-shot fast spin echo (SS-FSE) sequence with a smaller FOV (for example $18 \mathrm{~cm}$ ) was used to acquire more detailed sagittal images of the fetal brain (Fig. 6). Because the fetal head can be oriented in any direction within the mother's abdomen, a series of oblique SS-FSE sequences were often required before a satisfactory set of sagittal images was obtained. These sagittal images were then used to prescribe slice orientation for the subsequent acquisition of axial images.

\section{Relaxometry}

Relaxation times are sensitive to variations in the fundamental physical properties of tissue that are measurable with MRI. These properties include the water content of gray matter and white matter, which change throughout fetal and postnatal life. Relaxometry has the added value for longitudinal studies because its parameters can be held constant with repeated imaging over gestation, unlike $T_{1}$ - and $T_{2}$-weighted images, which require reoptimization at each point in development to compensate for age-related changes in relaxation times.

Relaxometry therefore was adapted to document changes in tissues of the fetal brain during development.

Our relaxometry protocol used fast spin-echo (FSE) sequences with high echo train lengths (ETL) to acquire $T_{1}$ and $T_{2}$ relaxation times in the fetal brain. Non-spin-echo sequences are prone to susceptibility-induced distortion artifacts, which increase greatly during in utero imaging, particularly at high field strengths. FSE sequences, in contrast, acquire multiple spin echoes in a single TR to accelerate image acquisition. FSE therefore relies on use of $T_{2^{-}}$ weighted, not $T_{2}{ }^{*}$-weighted, echo trains and is therefore less sensitive to susceptibility-induced distortion artifacts. The successful use of FSE imaging sequences to acquire relaxometry measures in the brains of newborn humans has been documented previously (Jones et al., 2004; Liu et al., 2006).

The following parameters were used for relaxometry in the fetal baboon: $F O V=12 \mathrm{~cm}$, matrix size $=128 \times 128$ (zero-filled to $256 \times 256$ ), slice thickness $=3 \mathrm{~mm}$, slice spacing $=0 \mathrm{~mm}, \mathrm{NEX}=1$, echo train length $(E T L)=32$. The no phase wrap $(\mathrm{NPW})$ option on the scanner was used because the size of the maternal abdomen was larger than the selected FOV $(12 \mathrm{~cm})$. These parameters were held constant in repeated studies across gestation. For $T_{2}$ measurements using an FSE sequence, images were acquired at effective echo times (TEs) of 10, 35, 70, 105, 140, 175, 210 , and $245 \mathrm{~ms}$, with a repetition time (TR) $=3500 \mathrm{~ms}$. Acquisition of 20 or more slices at all TEs required approximately $25 \mathrm{~min}$ for $T_{2}$ measurements. An inversion recovery (IR)-FSE sequence was used to acquire $T_{1}$ measurements at inversion times (TIs) of 60, 300, 750, 1500, and $3000 \mathrm{~ms}$, with a TR=10,000 $\mathrm{ms}$ and $\mathrm{TE} \approx 10 \mathrm{~ms}$. Acquisition of 20 or more slices required from 3 to 9 min, depending on the TI. The total acquisition time for $T_{1}$ measurements was about $25 \mathrm{~min}$. Maps of $T_{1}$ and $T_{2}$ values in the fetal brain were obtained by curve fitting on a pixel-by-pixel basis the series of $T_{1}$ - and $T_{2}$-weighted images obtained at differing TIs or TEs. A single component for $T_{1}$ and $T_{2}$ was assumed for the calculation of $T_{1}$ and $T_{2}$ values. Thus, the signals at differing TIs or TEs in the respective IR-FSE or FSE sequences could be characterized by the following equations:

$$
S=M_{0}\left|1-2 e^{-\mathrm{TI} / T_{1}}+e^{-\left(\mathrm{TR}-\mathrm{TE}_{\text {last }}\right) / T_{1}}\right| e^{-\mathrm{TE}_{\text {eff }} / T_{2}}(\mathrm{IR}-\mathrm{FSE}),
$$

Where $\mathrm{TE}_{\text {last }}=\mathrm{ETL} \times \mathrm{esp}$; 


$$
S=M_{0}\left(1-2 e^{-(\mathrm{TR}-\mathrm{TE} / 2) / T_{1}}+e^{-\mathrm{TR} / T_{1}}\right) e^{-\mathrm{TE} / T_{2}}(\mathrm{FSE}) .
$$

When TR $\gg$ TE, Eq. (2) assumes a simple mono-exponential relationship:

$$
S=S_{0} e^{-\mathrm{TE} / T_{2}}, \text { where } S_{0} \approx M_{0}\left(1-e^{-\mathrm{TR} / T_{1}}\right) .
$$

After obtaining $T_{1}$ and $T_{2}$ maps for each fetal baboon at multiple gestational ages (GAs), we calculated the average values of $T_{1}$ and $T_{2}$ in selected ROIs located in cortical gray matter (CGM) and in areas medial and adjacent to CGM, where white matter (WM) eventually develops (Fig. 7). These ROIs are positioned as consistently as possible across scans acquired at different GAs, but may not represent identical tissues and locations because of the rapid structural changes in the fetal brain with increasing GA. Nevertheless, these ROIs contained representative regions of CGM and the areas medial to it. The medially adjacent regions are designated white matter (WM), although this designation has not been documented with histological examinations. Given this assumption, the average values of $T_{1}$ and $T_{2}$ in the ROIs were used to represent the general characteristics of CGM and WM over the course of fetal brain development.

\section{$T_{1}$ - and $T_{2}$-weighted anatomical images}

$T_{1}$-weighted (T1W) and $T_{2}$-weighted (T2W) images of high resolution are useful for revealing detailed anatomical features of the fetal brain (Huisman et al., 2002). T1W images typically are used to study myelination, whereas $\mathrm{T} 2 \mathrm{~W}$ images typically are used to study regional anatomy in the fetal brain. $\mathrm{T} 2 \mathrm{~W}$ studies are more common because they require a shorter scan time and are less subject to artifacts. However, maternal or fetal motion and the tissue and chemical composition of the intrauterine environment reduce SNR and increase the likelihood of artifacts, such as motion and ghosting, in both T1W and T2W images. In clinical studies of the human fetus, MRI typically is performed using ultrafast $T_{2}$-weighted sequences, such as single-shot fast spin echo (SS-FSE), which produce a relatively low spatial resolution ( $1 \mathrm{~mm} \times 1$ $\mathrm{mm}$ in-plane resolution and a slice thickness of $4 \mathrm{~mm}$ or more) (Coakley et al., 2004; Huisman et al., 2002). Our goal was to develop techniques for acquiring T1W and T2W images that have a higher spatial resolution.

A series of sequences were evaluated and their parameters tuned to improve image resolution. For anatomical imaging, we aimed for a resolution between $0.5 \times 0.5 \times 1.0 \mathrm{~mm}^{3}$ to $1.0 \times 1.0 \times 1.0$ $\mathrm{mm}^{3}$. We initially used standard anatomical sequences, such as FSPGR and FLAIR. However, low SNR and large motion and ghosting artifacts prevented the acquisition of useful highresolution images with these sequences (Fig. 8).

Subsequently, we adopted the use of Fast Spin Echo (FSE) sequences to acquire T1W and T2W images of fetal brains. FSE allowed images with higher in-plane resolution (FOV of 12 $14 \mathrm{~cm}$ with a matrix more than $128 \times 128$, up to $192 \times 192$, all zero-filled to $256 \times 256$ ) and better through-plane resolution (slice thicknesses of $2.0-3.0 \mathrm{~mm}$, with $0 \mathrm{~mm}$ spacing). These images improved on the resolution of more standard anatomical sequences. For T2W images, sequence parameters included TR=5000 ms, TE=140 ms, ETL=32; for T1W images, TR=500 ms, $\mathrm{TE}=$ minimum (about $10 \mathrm{~ms}$ ), $\mathrm{ETL}=4$. The acquisition time to acquire 30 or more slices was approximately $5 \mathrm{~min}$ each for a single $\mathrm{T} 1 \mathrm{~W}$ or $\mathrm{T} 2 \mathrm{~W}$ scan. These sequences produced images with a resolution of $0.9 \times 0.9 \times 2 \mathrm{~mm}^{3}$ (Fig. 9).

\section{Diffusion tensor imaging}

DTI measures the trajectory of nerve fiber tracts primarily in white matter of the brain by measuring the diffusion of water molecules, which itself is determined by directional 
orientation of nerve fibers. DTI thus is a promising method for tracking the formation of white matter fibers during development (Huang et al., 2006; Neil et al., 2002). Apart from being used to track fibers, DTI also can provide information about other microstructural features of both cortical gray matter (McKinstry et al., 2002) and white matter (Huppi et al., 1998) in the developing brain. DTI also can be used to generate maps of the mean diffusivity (MD) of water in the brain and measures of fractional anisotropy (FA), which describe the degree of directional organization of tissues. Given its utility, we attempted to use DTI to monitor the development of fiber tracts and the structural organization of gray and white matter in the fetal brain.

We used a DTI sequence based on EPI for all scans. We initially attempted to obtain highresolution images: $F O V=12 \mathrm{~cm}$, matrix size $=128 \times 128$, slice thickness $=2 \mathrm{~mm}$, slice spacing $=0$ $\mathrm{mm}$, along with a $b$-value of $1000 \mathrm{~s} / \mathrm{mm}^{2}$ and 11 directions of diffusion gradients. However, the SNR of the diffusion-weighted images that we acquired using these parameters was unacceptably low. We ultimately lowered the target resolution for the images and used the following parameters: $\mathrm{FOV}=12 \mathrm{~cm}$, matrix size $=64 \times 64$, slice thickness $=2 \mathrm{~mm}$, slice spacing $=0$ $\mathrm{mm}, \mathrm{TE}=100 \mathrm{~ms}, \mathrm{TR}=8000 \mathrm{~ms}, \mathrm{NEX}=3$, and $b$-value $=1000 \mathrm{~s} / \mathrm{mm}^{2}$. The acquisition time was approximately $6 \mathrm{~min}$.

\section{Results}

\section{High-resolution anatomical images}

High-resolution T2W images were used to evaluate structural development of the fetal brain. These structures included the cortical plate (CP), subplate (SP), ventricular zone (VZ), germinal matrix (GM), and Sylvian fissure (Fig. 10). Among other events, the initiation of cortical gyration and sulcation, and the appearance of the subplate, are noted before $90 \mathrm{dg}$.

\section{Relaxometry}

We observed that relaxation times for both $T_{1}$ and $T_{2}$ in the fetal brain were shorter in CGM than in medially adjacent areas (Fig. 7, middle and right). Both $T_{1}$ and $T_{2}$ values decreased in CGM and in medial areas as the brain matures. This decrease, however, occurred at differing rates across each tissue type (Figs. 11 and 12). $T_{1}$ relaxation times eventually became comparable across GM and WM, as did $T_{2}$ relaxation times. In support of the validity of these techniques, relaxation times of CSF remained constant over gestation. The diminishing differences in relaxation times between GM and WM with advancing GA produced progressively poorer image contrast as the fetal brain matured. Indeed, the known effects of relaxation times on the tissue contrast $(C)$ between GM and WM in $T_{1}$ and $T_{2}$ maps indicate that the values of the contrasts $C_{\mathrm{T}_{1}}$ and $C_{\mathrm{T}_{2}}$ approach zero near term (Fig. 13):

$$
C_{T_{1}}=\frac{T_{1}^{\mathrm{WM}}-T_{1}^{\mathrm{GM}}}{T_{1}^{\mathrm{WM}}+T_{1}^{\mathrm{GM}}} \text {, and } C_{T_{2}}=\frac{T_{2}^{\mathrm{WM}}-T_{2}^{\mathrm{GM}}}{T_{2}^{\mathrm{WM}}+T_{2}^{\mathrm{GM}}},
$$

The absence of contrast was confirmed in the T1W and T2W images (Fig. 10). Relaxation times continued to decline after birth, and ultimately $T_{1}$ and $T_{2}$ values became higher for CGM than for WM in adulthood (Miot-Noirault et al., 1997). Thus the contrast of MR images for the brain of the postnatal infant is the reverse of that for the fetal brain (Fig. 14).

\section{Diffusion tensor imaging}

We used both the FACT (fiber assignment by continuous tracking) algorithm (Mori et al., 1999) and a revised algorithm based on FACT (Xu et al., 2002) for fiber tracking. We assessed various parameters for tract termination in both algorithms. However, fiber tracking could not be carried out successfully using any of the DT images that we acquired because of insufficient 
SNR in the diffusion-weighted images. Low SNR caused fiber tracking to terminate prematurely, hindering our efforts to track fibers using these algorithms. DTI nevertheless did provide useful information about early development in the fetal baboon brain. For example, no fiber tracts were detectable using DTI from roughly 60 to $80 \mathrm{dg}$. However, by approximately $110 \mathrm{dg}$, the corpus callosum was clearly visible (Fig. 15), and by $130 \mathrm{dg}$, tissue orientation with a radial organization was observed in the developing cortical mantle (Fig. 16). From 6 fetal DTI scans (mean gestational age $126 \pm 26 \mathrm{dg}$, range: 90 to $162 \mathrm{dg}$ ), the value of mean diffusivity was $1.81 \pm 0.15 \mu \mathrm{m}^{2} / \mathrm{ms}$ in white matter and $1.30 \pm 0.14 \mu \mathrm{m}^{2} / \mathrm{ms}$ in deep gray matter.

\section{Discussion}

We report the development of an experimental protocol for use of a 3T whole-body MRI scanner for imaging the brain of a nonhuman fetal primate. To develop this protocol, a series of MRI pulse sequences and procedures were evaluated in scanning 7 pregnant baboons. We found that a standard quadrature head coil is more suitable for imaging fetal baboons than is either a body coil or an enlarged head coil. Use of the quadrature coil improves SNR and accommodates the increasing girth of pregnant animals throughout gestation. We found that carefully calibrated and monitored inhalation anesthesia suppresses both maternal and fetal motion artifact to a substantial degree, making possible longer imaging sequences and repeated studies across time, without compromising the pregnancy. Standardization of pulse sequencing and imaging planes led to success in mapping $T_{1}$ and $T_{2}$ relaxation times for the entire brain sequentially throughout the latter $70 \%$ of term pregnancies. We consequently were able to record the average values of $T_{1}$ and $T_{2}$ in various brain tissues across fetal development. In addition, strategies adapted for fetal studies provided high-resolution T1W and T2W anatomical images. Both relaxation maps and anatomical images provided unique information about fetal brain development.

Implementation of the protocol required that specific technical issues be addressed. First, positioning of the fetal brain within the coil and the magnet, as well as accurate localization of the image slices, were all crucial to achieving good image quality. Second, wrap-around artifacts were recognized as a substantial challenge in fetal imaging, and their attenuation was achieved through use of the "no phase wrap" option on the scanner. Saturation bands were not used to suppress signals from outside of the selected FOV because we found that their use compromised SNR and image contrast. Our evaluation showed that the development of imaging sequences must include care to avoid exceeding the limitations of SAR that are programmed into commercial scanners. Scanners are programmed to terminate the scan automatically when a certain FDA-defined level of RF energy deposition has been exceeded. These interruptions degrade image quality. This problem was addressed by careful scheduling of RF-intense and non RF-intense sequences to reduce the cumulative deposition of RF energy.

In evaluating a series of strategies to track brain maturation in utero, we found that the acquisition of $T_{1}$ and $T_{2}$ maps of the entire fetal brain repeatedly across time was informative of changes in CGM and WM across time in various ROIs. We were unable to select identical ROIs across gestation because of the dramatic structural and volumetric changes that occur during fetal brain development. Thus, we opted to evaluate ROIs located in representative areas of CGM and WM, respectively. We then calculated the average values of $T_{1}$ and $T_{2}$ in these ROIs, and used these values to represent the general characteristics of changes in $T_{1}$ and $T_{2}$ relaxation during brain development.

We have shown that the average relaxation times $\left(T_{1}\right.$ or $\left.T_{2}\right)$ in CGM and WM decrease during the course of fetal development and converge near term. This finding confirms the implications of a previous study on postnatal baboons in which $T_{2}$ values in CGM and WM were similar 2 weeks after birth and then decreased continuously, each at differing rates, with $T_{2}$ values in 
WM becoming lower than those in GM near term (Miot-Noirault et al., 1997). Water in the brain exists in various forms, including water within myelin sheaths, in intra- and extracellular spaces, and in CSF (Whittall et al., 1991; Whittall and MacKay, 1989). The finding of continuously decreasing values of $T_{1}$ and $T_{2}$ during fetal development may be attributable to changes in the water content of the brain, and later to myelination in white matter (Counsell et al., 2003; Ferrie et al., 1999).

Documenting changes in $T_{1}$ and $T_{2}$ relaxation times across development also aids the effective application of other MRI modalities to fetal imaging, because knowledge of those values can be used to optimize the pulse sequences at various gestational ages. Without knowledge of $T_{1}$ and $T_{2}$ times in fetal brain at different time points in gestation, pulse sequence parameters, such as TR, TE, and TI, are typically decided based on the values of $T_{1}$ and $T_{2}$ in newborns and older infants, or even in adults, which likely will produce either suboptimal or grossly inadequate images.

Both the relaxation maps and the high-resolution anatomical images acquired in study of the fetal brain can be used to segment CGM and other brain tissues. The absolute value of the relaxation time at each pixel in a relaxation map reflects tissue properties $\left(T_{1}\right.$ or $\left.T_{2}\right)$ that are independent of specific pulse sequence parameters. For $T_{1}$ - and $T_{2}$-weighted images, in contrast, the grayscale value at each pixel depends not only on tissue properties, such as $T_{1}$ and $T_{2}$, but also on parameters of the pulse sequence, such as TR, TE, and TI. Therefore, relaxation maps arguably may be more suitable for segmentation of fetal brains across development than are $\mathrm{T} 1 \mathrm{~W}$ or $\mathrm{T} 2 \mathrm{~W}$ images, although substantiating this claim requires further study.

We successfully acquired data for both maps of relaxation times and anatomical images. Our attempts to acquire DTI data, however, were less successful. Our current technique for in utero DTI did not produce data that were sufficient for fiber tracking in the fetal brain, but it did yield useful measurements, such as FA, that may be informative of the progress of myelination and other features of cerebral organization. DTI has been used successfully to detect white matter tracts in human infants born prematurely (Partridge et al., 2005; Yoo et al., 2005), suggesting that in our study the smaller size and interference caused by the intrauterine environment may have been deciding factors in limiting data acquisition from the fetal baboon brain.

Future technological development should focus on tuning and modification of pulse sequences and hardware to improve further the quality of fetal MRI data. One important approach would be to design a multi-channel coil that can be used for parallel imaging in this experimental setting, which would simultaneously increase scan speed, decrease distortion artifacts, and reduce SAR. Future investigation also should address a number of methodological issues that pertain to scanning under anesthesia. For example, although we observed no harmful effects in baboon fetuses that were scanned for more than $3 \mathrm{~h}$ on repeated occasions, we have little data on the long-term effects on brain development that ensue from extensive MRI scanning under anesthesia. Understanding such effects, if they exist, would facilitate assessment of whether their potential influences affect the validity of findings from longitudinal imaging studies of nonhuman fetuses. In addition, future research should aim to measure regional variations of $T_{1}$ and $T_{2}$ values in CGM or WM. Measuring those regional variations may provide an effective tool for measuring differing rates of maturation across brain regions, although the validity of this approach should be confirmed through histological studies. 


\section{Conclusion}

We have developed a protocol for imaging the fetal brain in pregnant baboons at a field strength of $3 \mathrm{~T}$. Our protocol includes procedures for repeated studies in individual pregnancies across the latter $70 \%$ of gestation, and the reliable acquisition of both relaxation maps and highresolution anatomical images. The protocol, which included the optimization of imaging sequences and the use of anesthesia, produced images of high resolution and excellent quality. The study provides specifications to optimize the choice of radiofrequency coils, location of the imaging volume and slices, and parameters for pulse sequences. These procedures will advance longitudinal studies of brain maturation by allowing investigators to acquire more detailed information about the fetal brain at specific stages of development.

\section{Acknowledgements}

This work was supported in part by NIMH grants MH068318, K02-74677, and MH36197, NIDA grant DA017820, and the Suzanne Crosby Murphy Endowment at Columbia University. The authors thank Tung-Wah Kiu for animal care, Satie Shova for data acquisition, and Jason Royal for extensive editing of the manuscript.

\section{References}

Benes FM, Lange N. Two-dimensional versus three-dimensional cell counting: a practical perspective. Trends Neurosci 2001;24:11-17. [PubMed: 11163882]

Black KJ, Koller JM, Snyder AZ, Perlmutter JS. Atlas template images for nonhuman primate neuroimaging: baboon and macaque. Methods Enzymol 2004;385:91-102. [PubMed: 15130735]

Blinkov, SM.; Glezer, II. The Human Brain in Figures and Tables: a Quantitative Handbook. Plenum Press and Basic Books; New York: 1968.

Coakley FV, Glenn OA, Qayyum A, Barkovich AJ, Goldstein R, Filly RA. Fetal MRI: a developing technique for the developing patient. Am J Roentgenol 2004;182:243-252. [PubMed: 14684546]

Counsell SJ, Kennea NL, Herlihy AH, Allsop JM, Harrison MC, Cowan FM, Hajnal JV, Edwards B, Edwards AD, Rutherford MA. $T_{2}$ relaxation values in the developing preterm brain. Am J Neuroradiol 2003;24:1654-1660. [PubMed: 13679288]

Dawson G, Ashman SB, Carver LJ. The role of early experience in shaping behavioral and brain development and its implications for social policy. Dev Psychopathol 2000;12:695-712. [PubMed: 11202040]

Dekaban AS. Tables of cranial and orbital measurements, cranial volume, and derived indexes in males and females from 7 days to 20 years of age. Ann Neurol 1977;2:485-491. [PubMed: 617590]

Dobbing J, Sands J. Quantitative growth and development of human brain. Arch Dis Child 1973;48:757767. [PubMed: 4796010]

Elster AD. Questions and answers. Am J Roentgenol 1994;162:1493-1497. [PubMed: 8192041]

Ferrie JC, Barantin L, Saliba E, Akoka S, Tranquart F, Sirinelli D, Pourcelot L. MR assessment of the brain maturation during the perinatal period: quantitative $T_{2}$ MR study in premature newborns. Magn Reson Imaging 1999;17:1275-1288. [PubMed: 10576713]

Hand JW, Li Y, Thomas EL, Rutherford MA, Hajnal JV. Prediction of specific absorption rate in mother and fetus associated with MRI examinations during pregnancy. Magn Reson Med 2006;55:883-893. [PubMed: 16508913]

Huang H, Zhang J, Wakana S, Zhang W, Ren T, Richards LJ, Yarowsky P, Donohue P, Graham E, van Zijl PCM, Mori S. White and gray matter development in human fetal, newborn and pediatric brains. NeuroImage 2006;33:27-38. [PubMed: 16905335]

Huisman TA, Martin E, Kubik-Huch R, Marincek B. Fetal magnetic resonance imaging of the brain: technical considerations and normal brain development. Eur Radiol 2002;12:1941-1951. [PubMed: 12136312]

Huppi PS, Maier SE, Peled S, Zientara GP, Barnes PD, Jolesz FA, Volpe JJ. Microstructural development of human newborn cerebral white matter assessed in vivo by diffusion tensor magnetic resonance imaging. Pediatr Res 1998;44:584-590. [PubMed: 9773850] 
Huttenlocher PR. Synaptic density in human frontal cortex - developmental changes and effects of aging. Brain Res 1979;163:195-205. [PubMed: 427544]

Huttenlocher PR. Morphometric study of human cerebral cortex development. Neuropsychologia 1990;28:517-527. [PubMed: 2203993]

Huttenlocher PR, Dabholkar AS. Regional differences in synaptogenesis in human cerebral cortex. J Comp Neurol 1997;387:167-178. [PubMed: 9336221]

Inder TE, Huppi PS. In vivo studies of brain development by magnetic resonance techniques. Ment Retard Dev Disabil Res Rev 2000;6:59-67. [PubMed: 10899798]

Jones RA, Palasis S, Grattan-Smith JD. MRI of the neonatal brain: optimization of spin-echo parameters. Am J Roentgenol 2004;182:367-372. [PubMed: 14736664]

Kraemer HC, Taylor YJ, Kupfer JL. How can we learn about development processes from cross-sectional studies, or can we? Am J Psychiatry 2000;157:163-171. [PubMed: 10671382]

Levitt P. Structural and functional maturation of the developing primate brain. J Pediatr 2003;143:S35S45. [PubMed: 14597912]

Lewis DA. The human brain revisited: opportunities and challenges in postmortem studies of psychiatric disorders. Neuropsychopharmacology 2002;26:143-154. [PubMed: 11790510]

Liu, F.; Peterson, BS.; Duan, Y.; Kangarlu, A. Fast spin echo for $T_{2}$ quantification at 3 T. The 14th Annual Meeting of ISMRM; Seattle. 2006. p. 2404

McKinstry RC, Mathur A, Miller JH, Ozcan A, Snyder AZ, Schefft GL, Almli CR, Shiran SI, Conturo TE, Neil JJ. Radial organization of developing preterm human cerebral cortex revealed by noninvasive water diffusion anisotropy MRI. Cereb Cortex 2002;12:1237-1243. [PubMed: 12427675]

Miot-Noirault E, Barantin L, Akoka S, Le Pape A. T2 relaxation time as a marker of brain myelination: experimental MR study in two neonatal animal models. J Neurosci Methods 1997;72:5-14. [PubMed: 9128162]

Mori S, Crain BJ, Chacko VP, van Zijl PC. Three-dimensional tracking of axonal projections in the brain by magnetic resonance imaging. Ann Neurol 1999;45:265-269. [PubMed: 9989633]

Myers C, Duncan KR, Gowland PA, Johnson IR, Baker PN. Failure to detect intrauterine growth restriction following in utero exposure to MRI. Br J Radiol 1998;71:549-551. [PubMed: 9691901]

Neil J, Miller J, Mukherjee P, Huppi PS. Diffusion tensor imaging of normal and injured developing human brain - a technical review. NMR Biomed 2002;15:543-552. [PubMed: 12489100]

Partridge S, Mukherjee P, Berman JI, Henry RG, Miller SP, Lu Y, Glenn OA, Ferriero DM, Barkovich AJ, Vigneron DB. Tractography-based quantitation of diffusion tensor imaging parameters in white matter tracts of preterm newborns. J Magn Reson Imaging 2005;22:467-474. [PubMed: 16161075]

Prayer D, Kasprian G, Krampl E, Ulm B, Witzani L, Prayer L, Brugger PC. MRI of normal fetal brain development. Eur J Radiol 2006;57:199-216. [PubMed: 16413984]

West MJ. Stereological methods for estimating the total number of neurons and synapses: issues of precision and bias. Trends Neurosci 1999;22:51-61. [PubMed: 10092043]

Whittall KP, MacKay AL. Quantitative interpretation of NMR relaxation data. J Magn Reson 1989;84:134-152.

Whittall KP, Bronskill MJ, Henkleman RM. Investigation of analysis techniques for complicated NMR relaxation data. J Magn Reson 1991;95:221-234.

Xu D, Mori S, Solaiyappan M, van Zijl PCM, Davatzikos C. A framework for callosal fiber distribution analysis. NeuroImage 2002;17:1131-1143. [PubMed: 12414255]

Yoo S-S, Park H-J, Soul JS, Mamata H, Park H-W, Westin C-F, Bassan H, Du Plessis AJ, Robertson RL, Maier SE, Ringer SA, Volpe JJ, Zientara GP. In vivo visualization of white matter fiber tracts of preterm- and term-infant brains with diffusion tensor magnetic resonance imaging. Invest Radiol 2005;40:110-115. [PubMed: 15654256] 


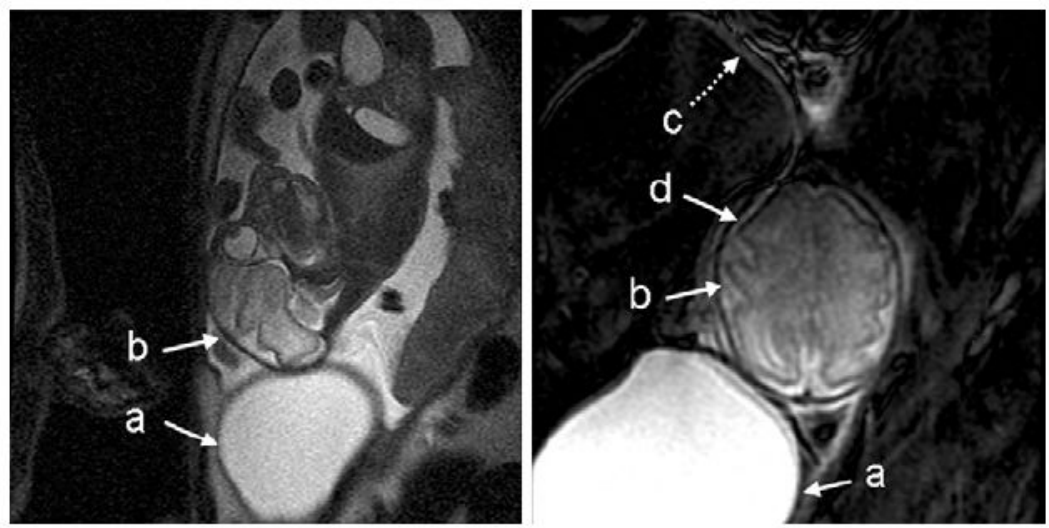

Fig. 1.

Intensity and motion artifacts. Both images show signal inhomogeneity caused by the full bladder (a). The image on the right shows motion artifact through the fetal brain (b) and ghosting of the bladder wall (c) that overlaps the periphery of the brain (d). Left: The fetus is shown in parasagittal cross-section in the head-down position. Right: The fetal brain is visible in transaxial cross-section. 


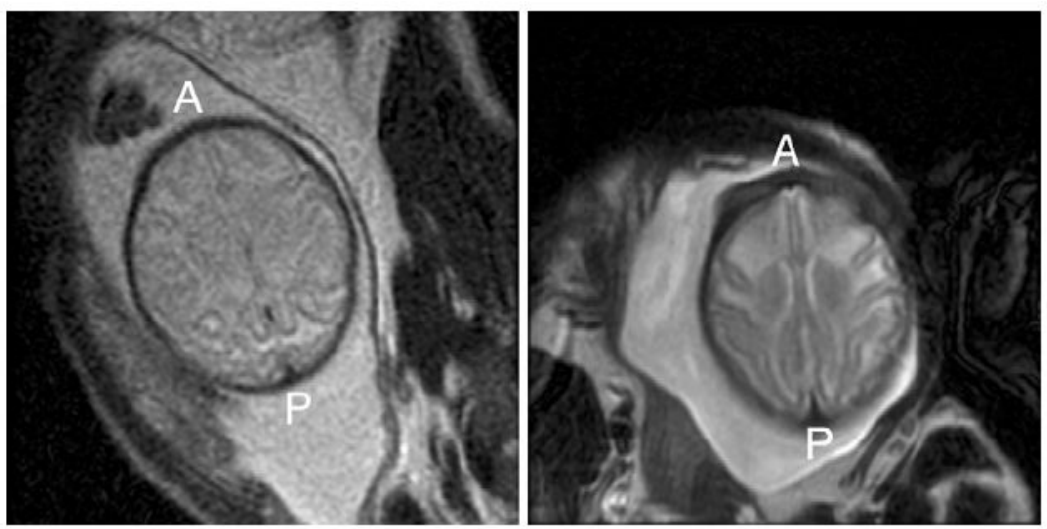

Fig. 2.

Body vs. head coil. Fast spin echo (FSE) images. Left: Acquired using a GE body coil at 154 dg. Right: Acquired using a GE quadrature head coil at $153 \mathrm{dg}$. FOV=12 cm, matrix $=128 \times 128$, $3 \mathrm{~mm}$ slice thickness is identical in each. The SNR of the image acquired using the head coil is substantially better than the image acquired using the body coil. Both images are in the transaxial orientation acquired in differing animals. A: anterior; P: posterior. 

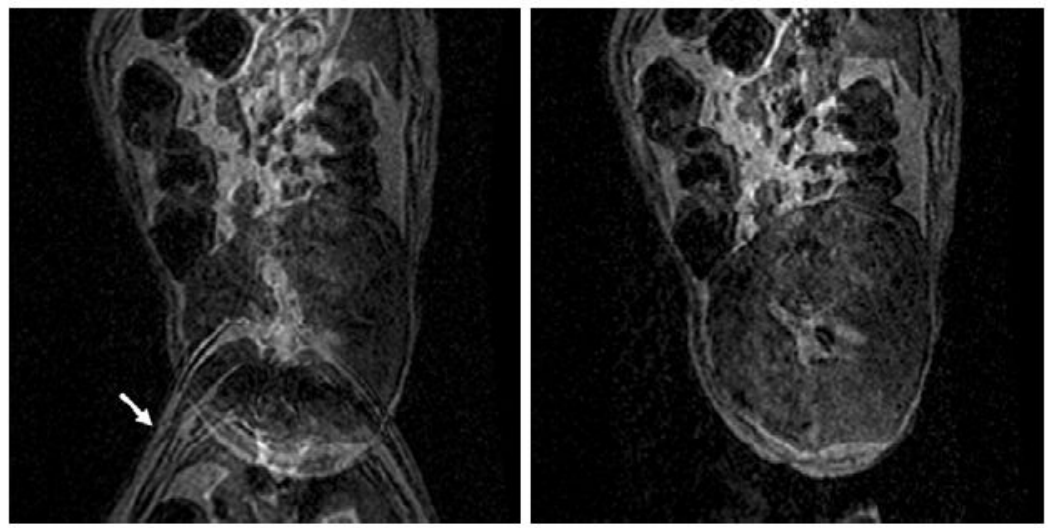

Fig. 3.

Wrap-around artifact. Left: Image of the maternal abdominal cavity acquired using an RF body coil without use of the no-phase wrap (NPW) option. Right: Image acquired with the same coil but with use of the NPW option. The wrap-around artifact without use of NPW (arrow) is greatly reduced. The fetal brain is not visible in either image. 

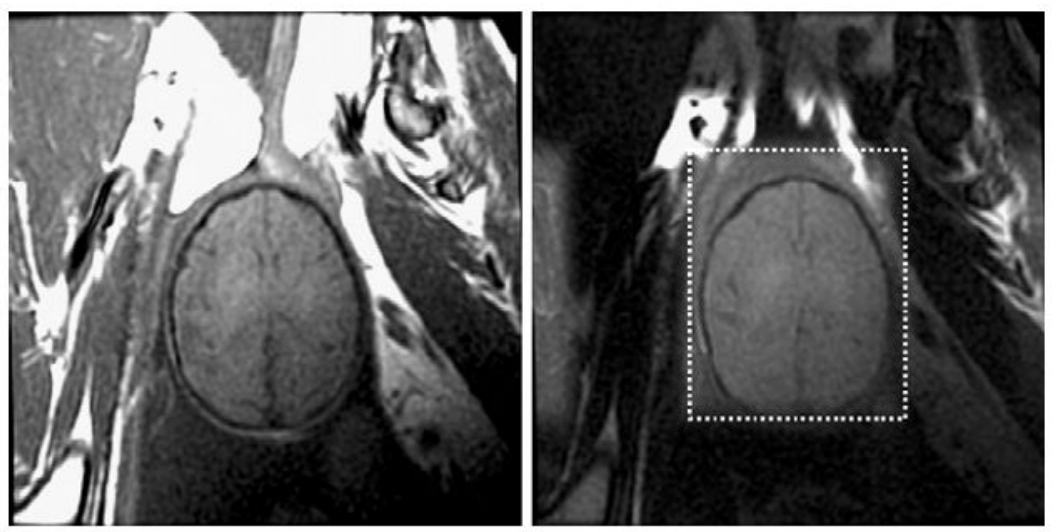

Fig. 4.

Saturation bands. These are $T_{1}$-weighted images of the same fetal brain in transaxial views, with the frontal cortex positioned superiorly in the image; $F O V=14 \mathrm{~cm}$, matrix $=128 \times 128$, $\mathrm{NEX}=2$. Left: Without use of saturation bands, intensity inhomogeneities are evident within the brain, which originate from fat and other tissues outside of the brain. Right: With use of saturation bands, inhomogeneities are reduced, but SNR and contrast within the FOV defined by the saturation bands (dotted rectangle) are reduced. 

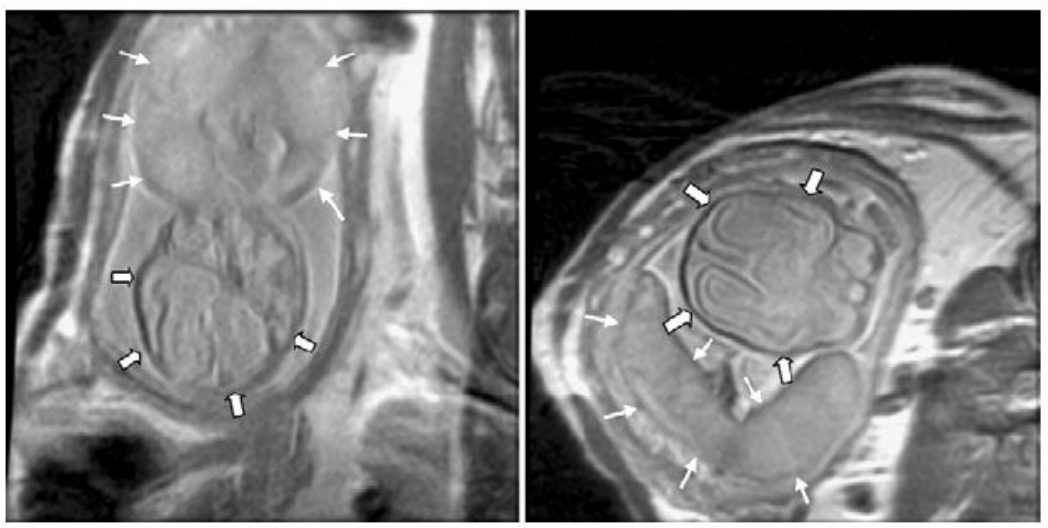

Fig. 5.

Reducing motion artifact. These are images from the same fetal brain before and after the fetal head was repositioned and the depth of anesthesia was optimized. FOV $=12 \mathrm{~cm}$, matrix $=128 \times 128, N E X=1$. Left: Image affected by motion artifact caused by the position of fetal head (block arrows) against the placenta (line arrows). Right: Motion artifact is substantially reduced by repositioning of the fetal head and by adjusting the depth of anesthesia. 

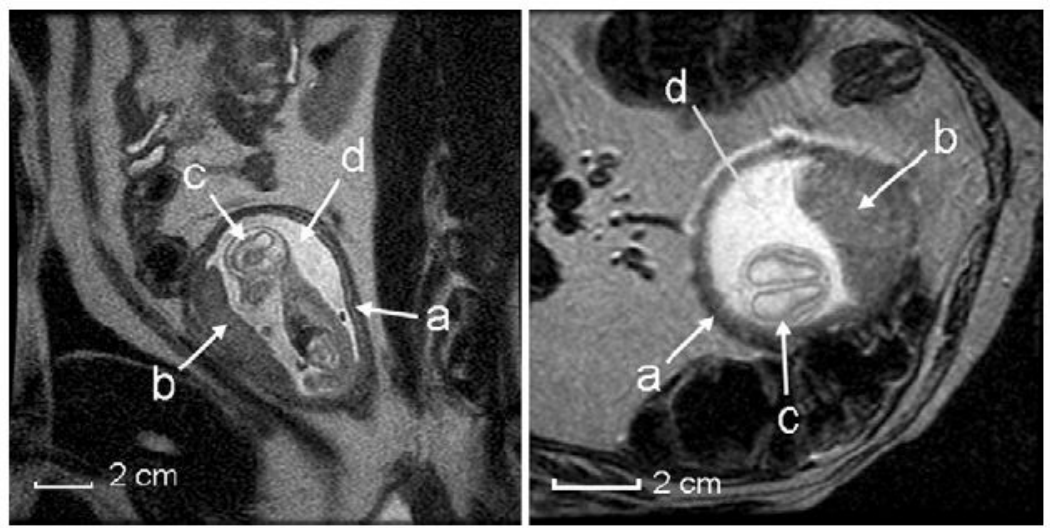

Fig. 6.

Slice positioning. Left: SS-FSE image for localization: a baboon fetus at $64 \mathrm{dg}$. FOV=18 cm, matrix $=288 \times 192$ (zero-padded to $512 \times 512$ ), $3 \mathrm{~mm}$ slice thickness. Shown is a parasagittal section of the leftward-facing fetal brain and torso, which is most helpful in prescribing the transaxial sections most useful for anatomical localization. Right: A transaxial $T_{2}$-weighted FSE image acquired from the same fetus. FOV $=12 \mathrm{~cm}$, matrix $=192 \times 160$ (zero-padded to $256 \times 256), 3 \mathrm{~mm}$ thickness, TE=136 ms, TR=3500 ms. At this early stage of development, few landmarks in the brain are distinguishable for slice localization. The ventricles are large relative to overall cerebral volume ("physiological fetal hydrocephalus"), and the cortex is minimally developed. $a=$ uterus, $b=$ placenta, $c=$ fetal brain, $d=$ amniotic fluid. 

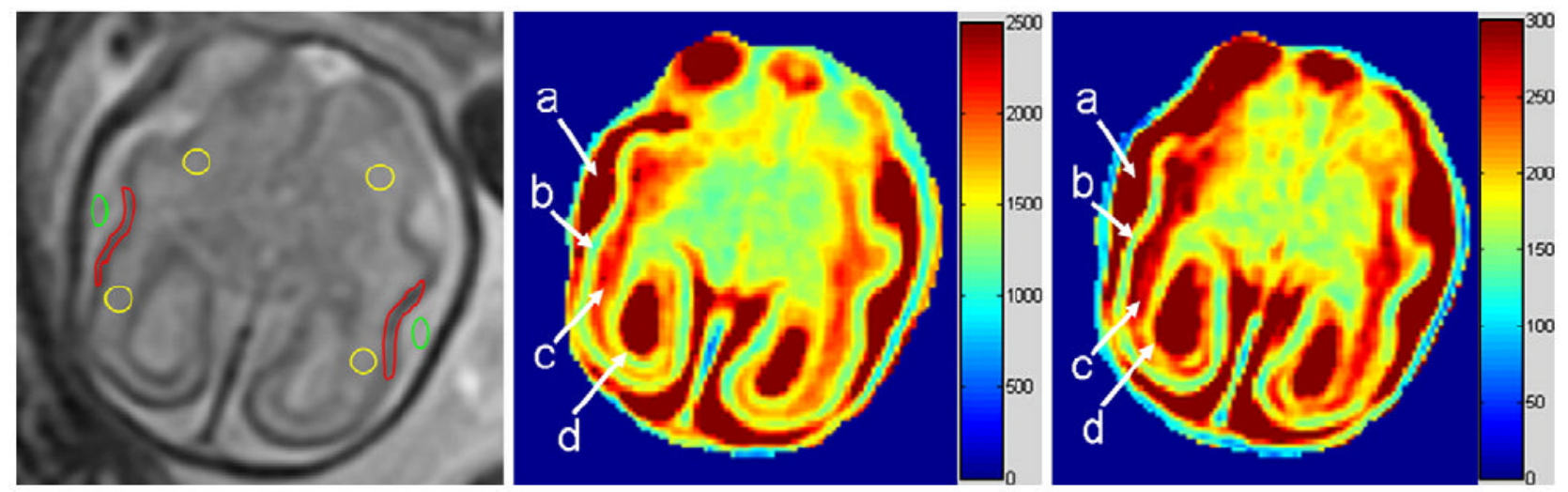

Fig. 7.

Maps of relaxation times. The brain of a fetal baboon (animal \#415—see Figs. 12, 13) at 105 $\mathrm{dg}$. Left: $T_{2}$-weighted transaxial image with frontal cortex at the top of the image. Yellow circle is where white matter was sampled; red outline is where gray matter was sampled; and green is where CSF was sampled. Middle: Color-coded map of $T_{1}$ relaxation times (colors are encoded such that long $T_{1}$ times, reflecting in part greater water content, are represented in "hot" colors and short $T_{1}$ times, reflecting in part greater tissue structure, are represented in "cooler" colors). Right: Color-coded map of $T_{2}$ relaxation times (colors are encoded such that long $T_{2}$ times, reflecting in part greater water content, are represented in "hot" colors and short $T_{2}$ times, reflecting in part greater tissue structure, are represented in "cooler" colors). These maps demonstrate that relaxation times can provide considerably better contrast of tissues than do weighted images, and may prove extremely useful for segmentation of the image into differing types of tissue. The improved contrast over weighted images and its reproducibility is evident in maps of both $T_{1}$ and $T_{2}$ relaxation times, despite the fact that these relaxation times represent fundamentally different properties of the tissues. These relaxation times in fact represent invariant, quantitative properties of the tissues, unlike the gray scale values and contrast that are present in weighted images, which represent only qualitative differences in tissue characteristics. $\mathrm{a}=\mathrm{CSF}, \mathrm{b}=$ cortical plate, $\mathrm{c}=$ subplate, $\mathrm{d}=$ =ventricular zone. 

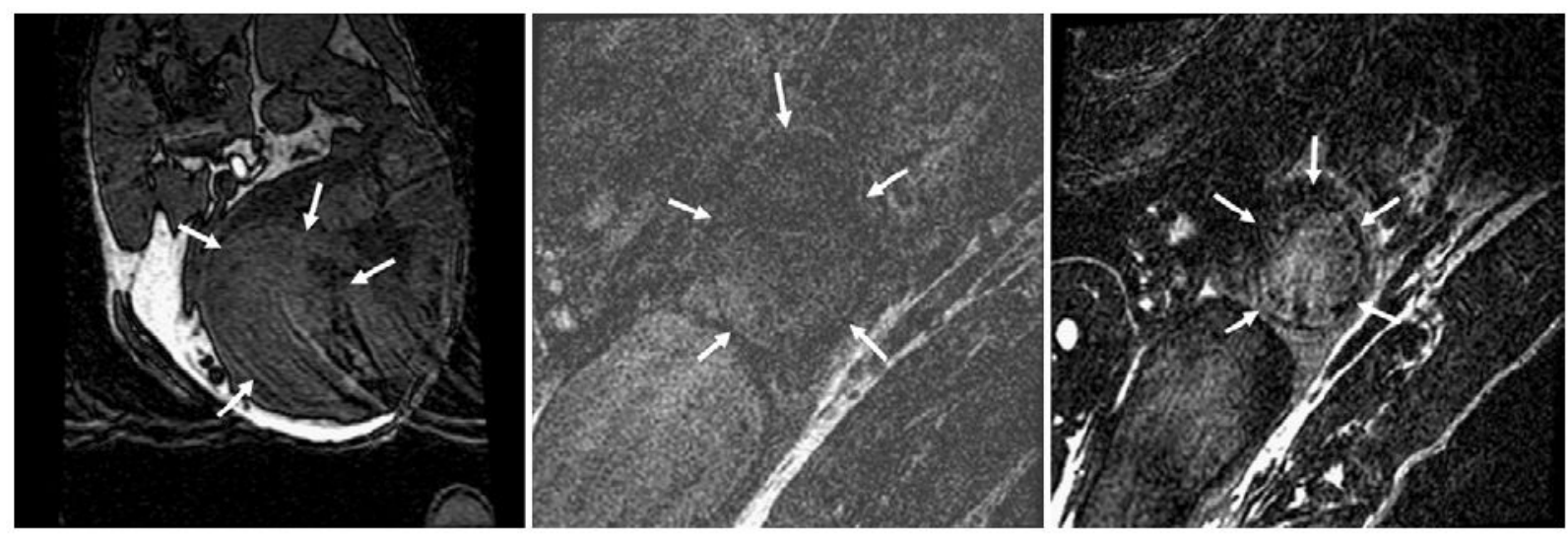

Fig. 8.

FSPGR and FLAIR images. Left: $T_{1}$-weighted FSPGR image, FOV=19 cm, matrix $=256 \times 128$, $1 \mathrm{~mm}$ slice thickness. Middle: Transaxial $T_{1}$-weighted FLAIR image, FOV=14 cm, matrix $=192 \times 128,1 \mathrm{~mm}$ slice thickness. Right: Transaxial $T_{2}$-weighted FLAIR image, FOV=20 cm, matrix 192×192, $1 \mathrm{~mm}$ slice thickness. In utero MR images of higher resolution typically are accompanied by poor SNR and prominent artifacts. The fetal brain (arrows) is barely visible, and differing brain structures cannot be discerned. 


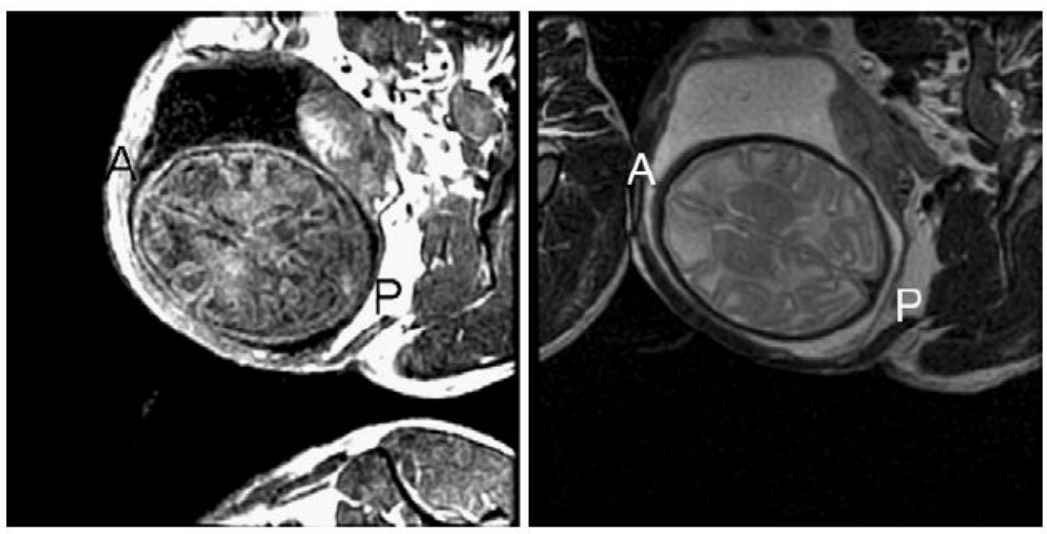

Fig. 9.

High-resolution, FSE images. $T_{1}$-weighted and $T_{2}$-weighted images of a fetal brain at $153 \mathrm{dg}$. Left: $T_{1}$-weighted FSE images with relatively high resolution $\left(0.9 \times 0.9 \times 2 \mathrm{~mm}^{3}\right)$. Right: $T_{2^{-}}$ weighted FSE images with the same resolution. FOV $=14 \mathrm{~cm}, 160 \times 160,2.0 \mathrm{~mm}$ slice thickness. With the help of anesthesia, FSE is an effective sequence to acquire $T_{1}$-weighted and $T_{2^{-}}$ weighted images of high resolution. $T_{2}$-weighed images provide much better contrast at this gestational age. A: anterior; P: posterior. 

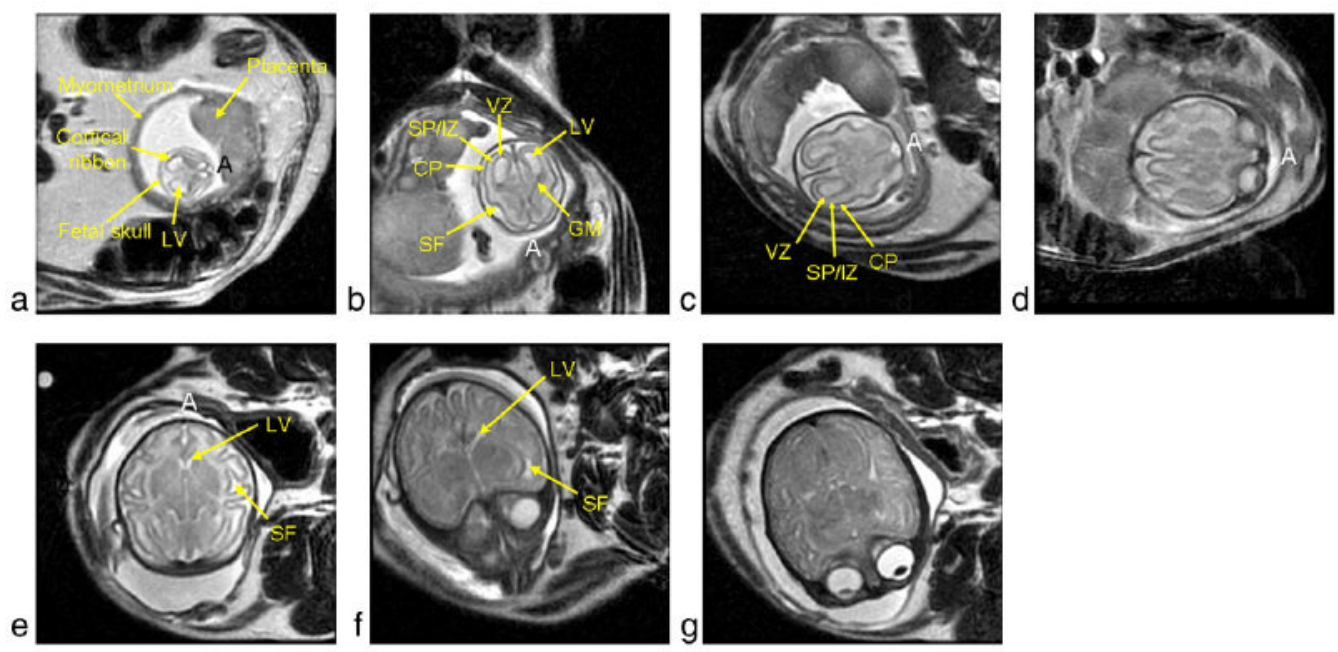

Fig. 10.

$T_{2}$-weighted images throughout the course of gestation. Examples of $T_{2}$-weighted FSE images of the fetal brain in transaxial views at various gestational ages: (a) At $64 \mathrm{dg}$, the large lateral ventricles $(\mathrm{LV})$ and the cortical ribbon dominate the brain. Other major structures are less well identified. The cortical surface of the frontal and occipital lobes are completely smooth, and sulci are not visible. (b) At $90 \mathrm{dg}$, multiple layers - the cortical plate (CP), subplate (SP), intermediate zone (IZ), and ventricular zone (VZ) - are visible in addition to the lateral ventricles. Cortical folding has begun. The Sylvian fissures (SF) are shallow but clearly visible. The germinal matrix (GM) is evident. (c) At $105 \mathrm{dg}$, the subplate is distinct and the Sylvian fissure and sulci are deepening. (d) At $132 \mathrm{dg}$, the cortex folds, sulci are more pronounced, and the subplate has regressed. (e) At $141 \mathrm{dg}$, the frontal and occipital cortices are folded and richly sulcated. The opening of the Sylvian fissure is nearly closed. (f) At $162 \mathrm{dg}$, the sulci are deeper and the Sylvian fissure is enclosed. (g) At $185 \mathrm{dg}$, the image contrast between gray matter and white matter is poor, a consequence of similar relaxation times in each tissue type. 

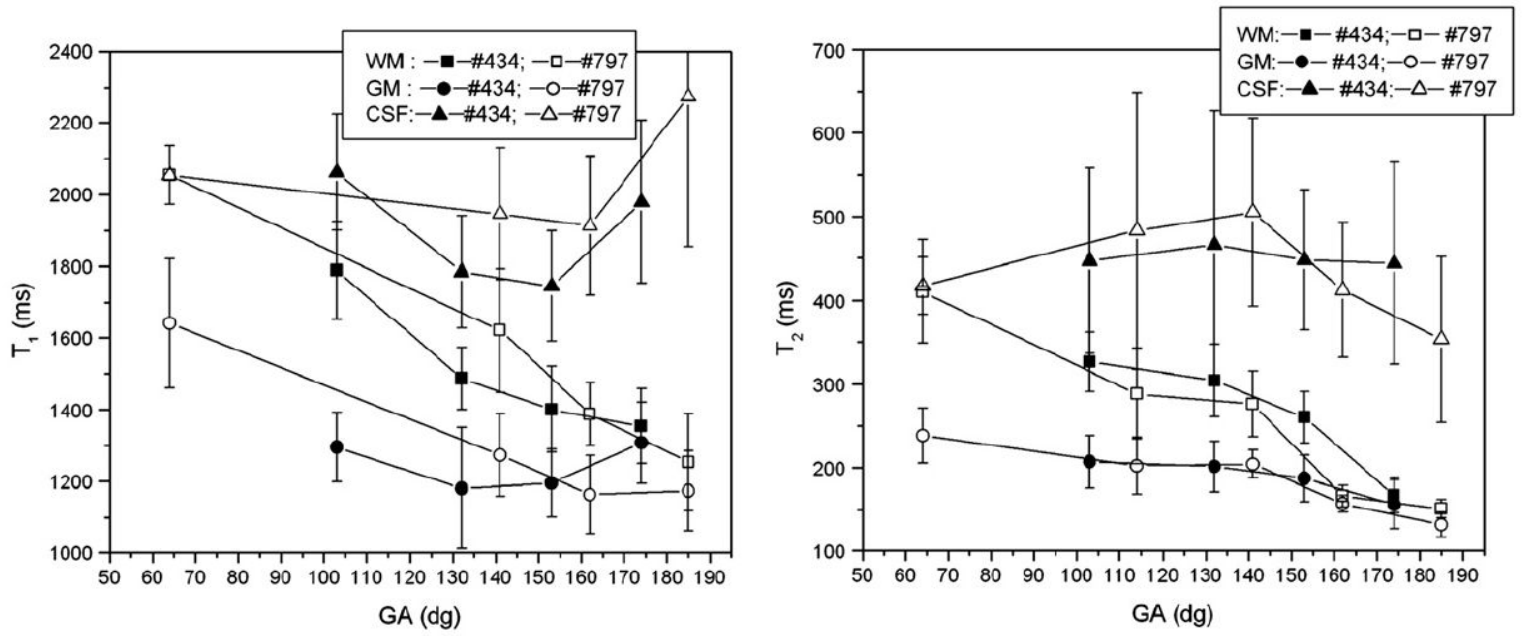

Fig. 11.

Longitudinal changes of relaxation times. Left: $T_{1}$ values for white matter (WM), gray matter (GM), and CSF with advancing gestational age (GA). Right: $T_{2}$ values with advancing GA. Linked data points are from individual fetuses (\#434 and \#797). Although the relaxation times decrease at different rates in GM and WM, they reach comparable values near term. 

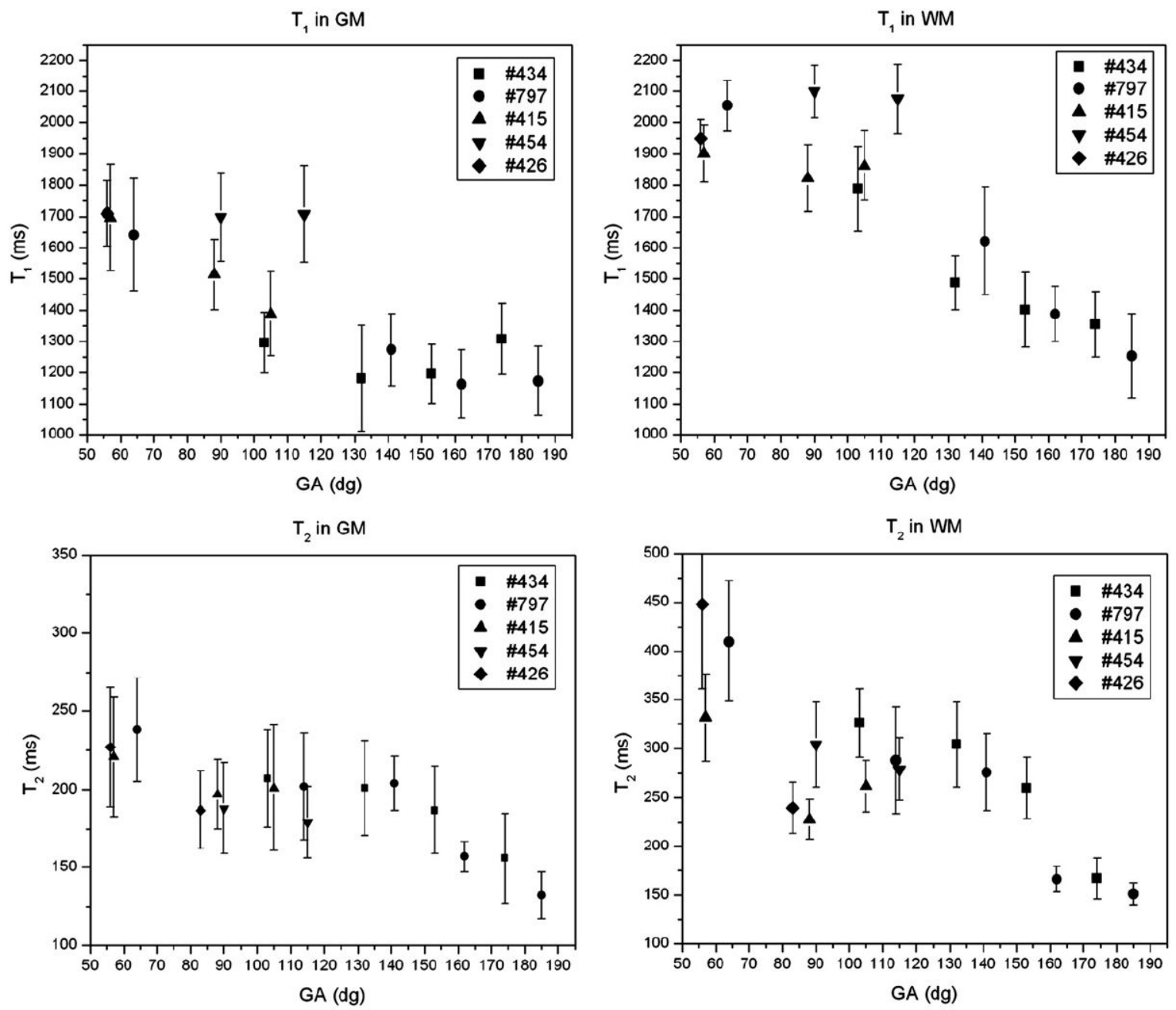

Fig. 12.

Relaxation times in CGM and WM. Left: Values of $T_{1}$ and $T_{2}$ in selected ROIs within CGM from 5 baboon pregnancies. Right: Values of $T_{1}$ and $T_{2}$ in selected ROIs within WM from the same baboons. The relaxation times in WM decrease at a faster rate than in CGM, becoming comparable to those in GM near term, and accounting for the absence of contrast across tissue types near that point in gestation. 


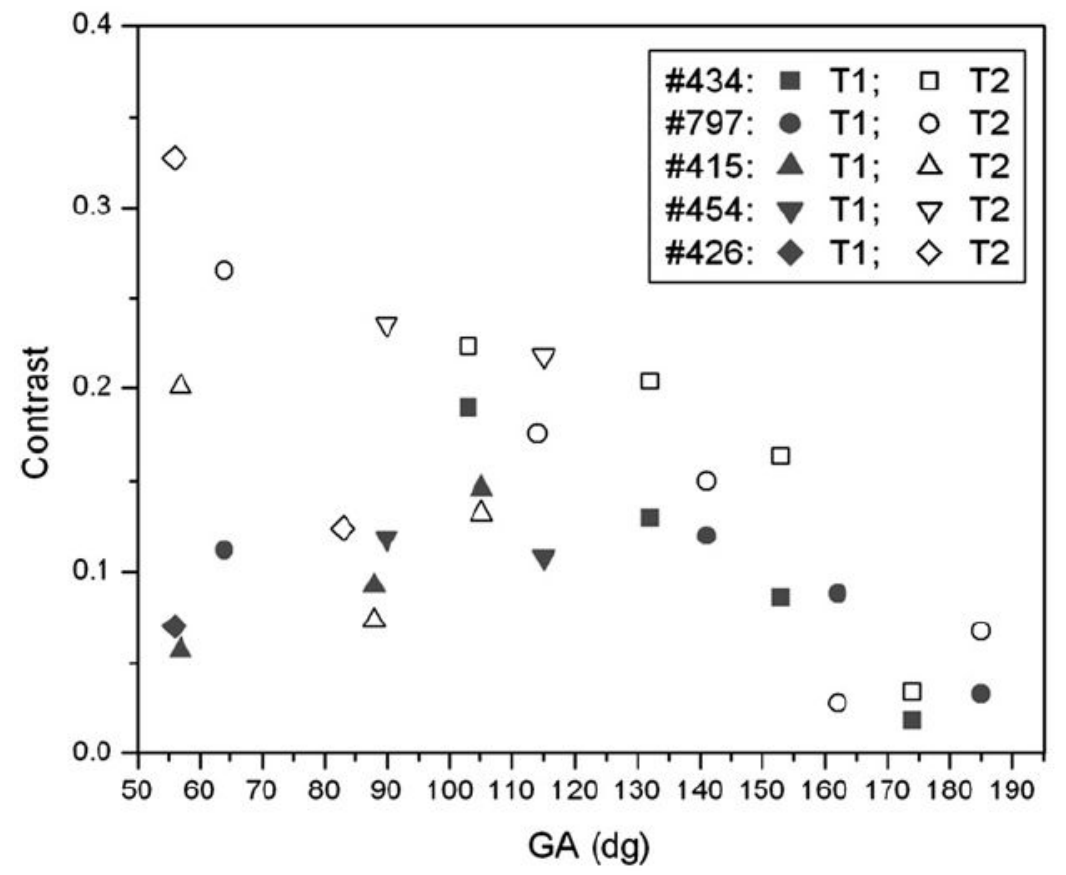

Fig. 13.

Contrast in maps of relaxation times. Contrast between CGM and WM in the maps of $T_{1}$ and $T_{2}$ relaxation times. Maximal contrast in $T_{2}$ maps is present earlier in gestation (e.g. before 80 $\mathrm{dg}$ ), whereas contrast within $T_{1}$ maps is maximal between 100 and $140 \mathrm{dg}$. Before $140 \mathrm{dg}$, tissue contrast in $T_{2}$ maps is superior to that in $T_{1}$ maps. The contrast in both $T_{1}$ and $T_{2}$ maps decreases to a minimum near term, where it is nearly negligible for both maps. 

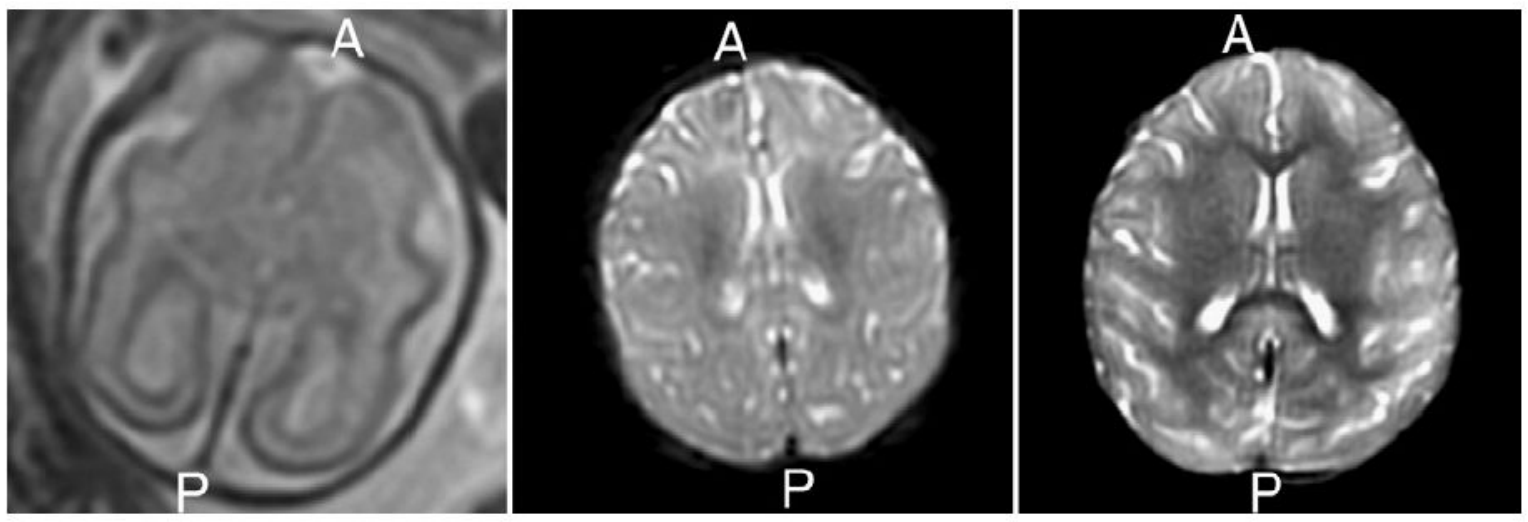

Fig. 14.

Evolution of image contrast. T2W FSE images of the same baboon. Left: T2W image at 105 $\mathrm{dg}$, in which gray matter (GM) appears darker than white matter (WM). Middle: T2W image 1 month after birth, in which the contrast between GM and WM is poor. Right: T2W image at 5 months after birth, in which WM appears darker than GM. Comparison of image contrast between GM and WM reveals a reversal of $T_{2}$ contrast during the course of fetal brain development. A: anterior; P: posterior. 

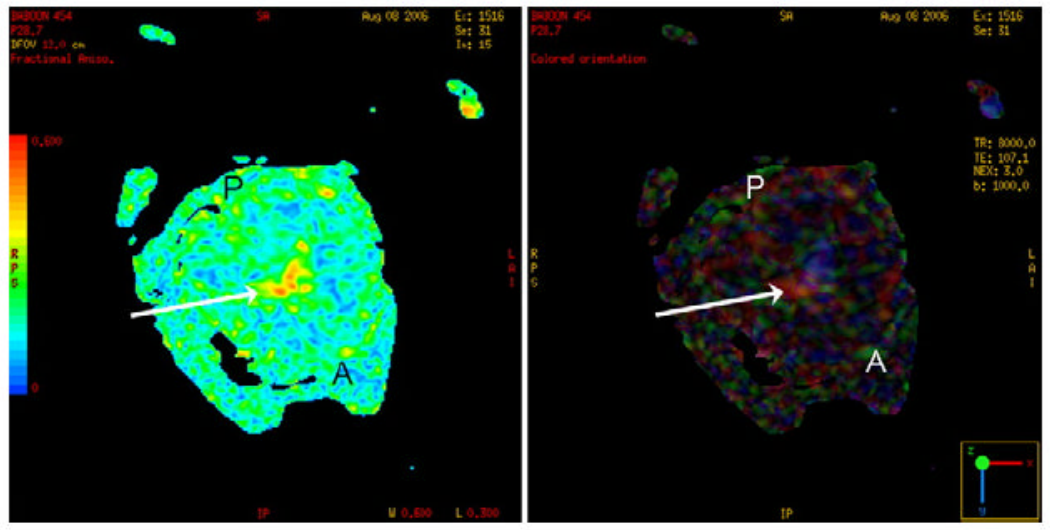

Fig. 15.

Fractional anisotropy and orientation maps. DTI maps of a fetal brain in transaxial views at $115 \mathrm{dg}$. Left: Map of fractional anisotropy (FA) (colors are encoded such that larger FA values are represented in "hot" colors, and smaller FA values are represented in "cooler" colors).

Right: Tensor map using colors to encode tensor orientation [ $x$-axis (red), $y$-axis (blue), $z$-axis (green) $. \mathrm{FOV}=12 \mathrm{~cm}$, matrix $=64 \times 64,2 \mathrm{~mm}$ slice thickness, $b$-value $=1000 \mathrm{~s} / \mathrm{mm}^{2}, 11$ gradient directions, $\mathrm{TR}=8000 \mathrm{~ms}, \mathrm{TE} \approx 100 \mathrm{~ms}$. FA and orientation maps can describe directional organization of brain tissues. By $115 \mathrm{dg}$, the corpus callosum (arrows) is already visible. A: anterior; P: posterior. 

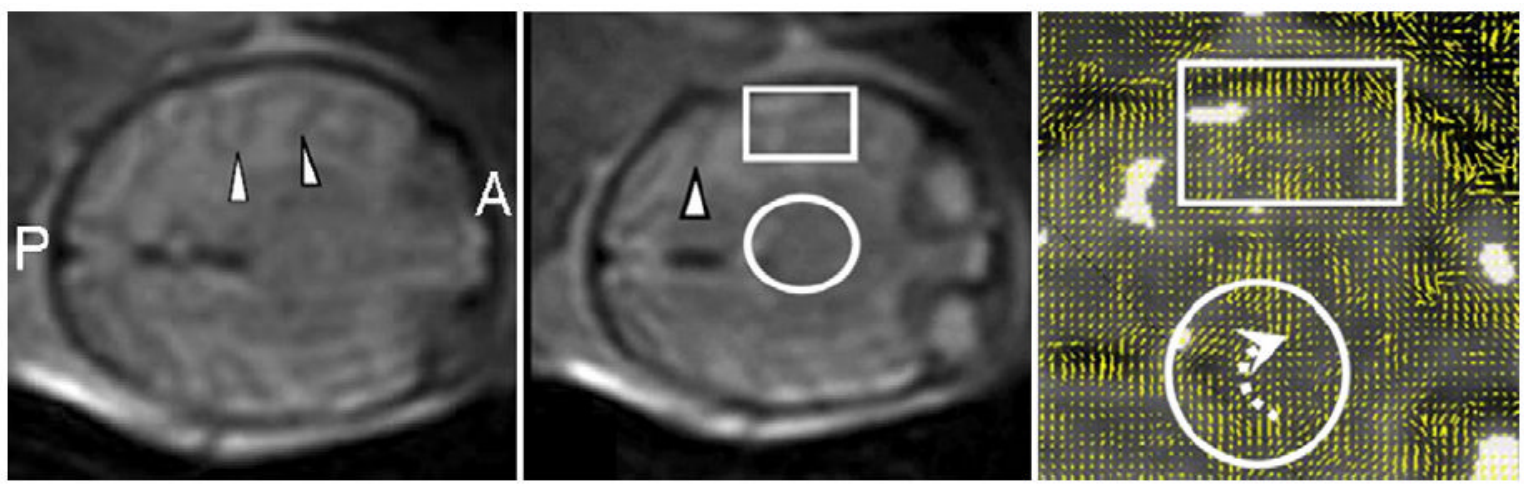

Fig. 16.

Trace and principal direction maps. DTI maps of a fetal baboon brain in transaxial views at $132 \mathrm{dg}$. Left: Trace maps. Middle: Trace maps of another slice from the same brain. Right: Principal direction map. The black band (arrowheads) indicates the cortical subplate. The rectangles indicate regions in which the axonal fibers are visible as dark lines. In the cortex, a radial organization is apparent, likely reflecting the presence of radial glia and possibly the prominent apical dendrites of developing pyramidal cells. In the white circles, non-radial, intracortical axons are visible. The dashed line in the right figure indicates the pathway of these intra-cortical fibers. A: anterior; P: posterior. 


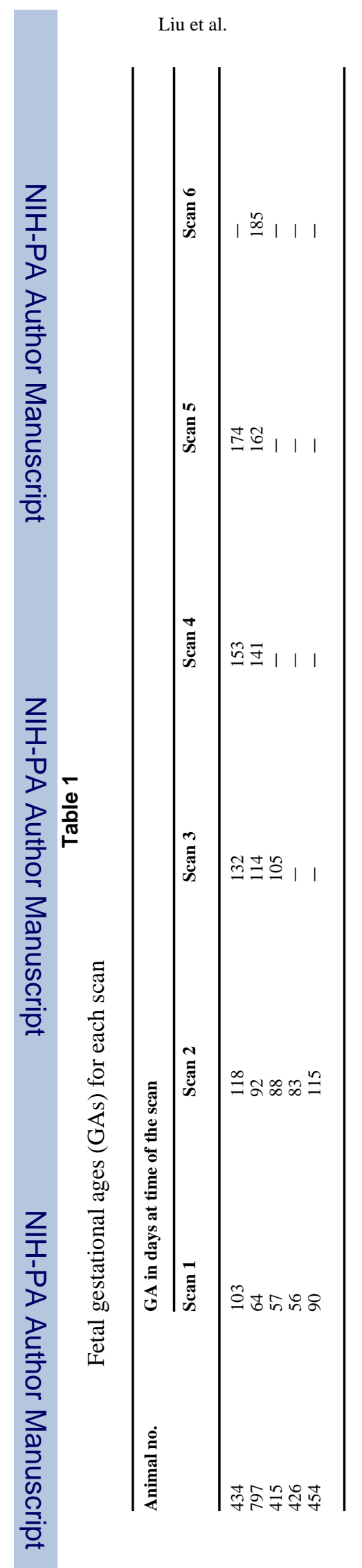

Neuroimage. Author manuscript; available in PMC 2009 March 1. 